
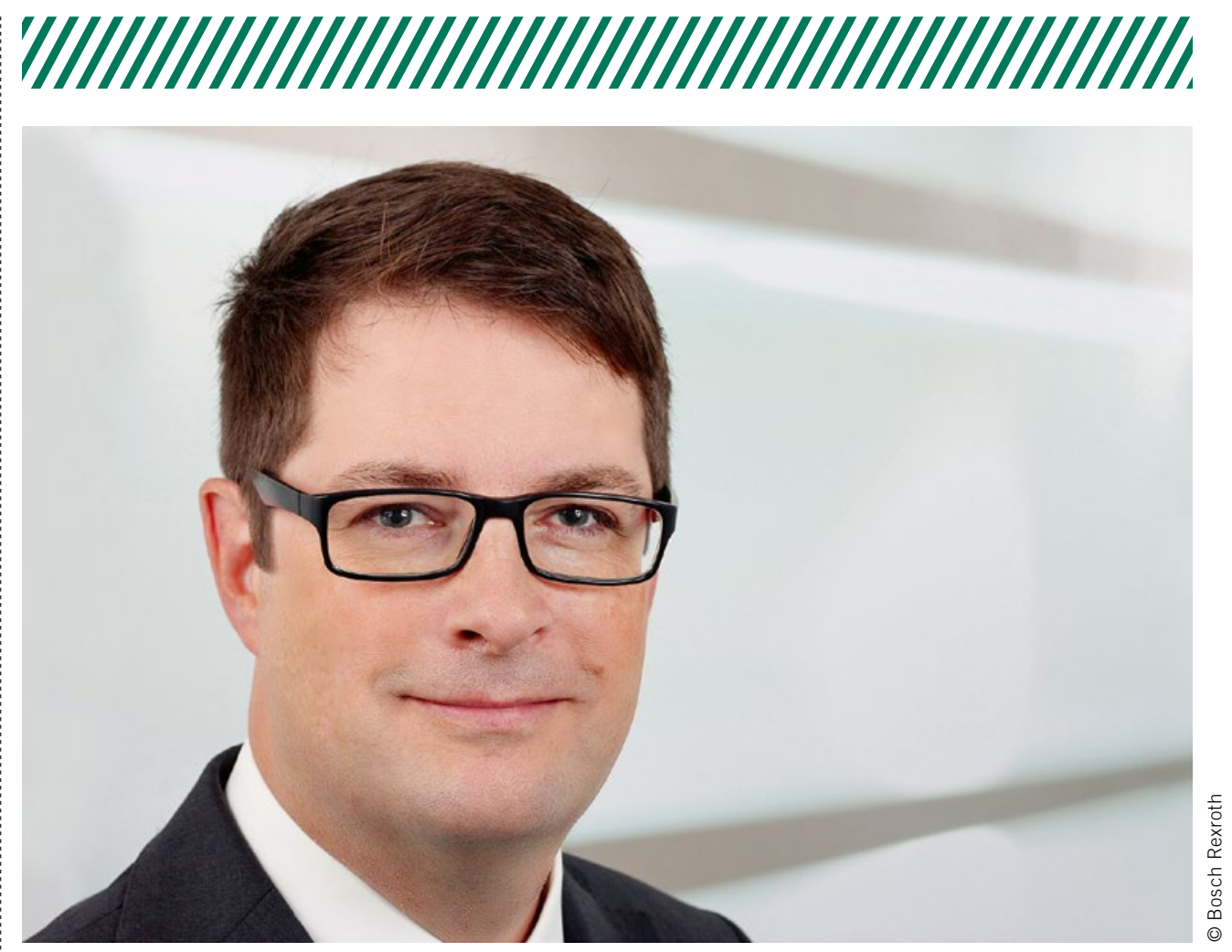

Dr. Alexander Flaig

Head of Engineering Mobile Electronics and Systems at the Bosch Rexroth AG in Elchingen (Germany)

\title{
Trend towards Electronification in the Construction Machinery Industry
}

For many years, there has been a trend towards the electronification of previously mechanical or hydraulic functions in agricultural machinery. Two main driving forces behind this are the adaptability of the tractor to carry out diverse work tasks as a universal machine and the pressure of improved energy efficiency (fuel costs are among the highest cost blocks in agriculture). In more recent times, the automation and networking of the work process chains has led to an increase in productivity.

Also in construction machines, low fuel consumption and engines that are as compact as possible are important factors. To operate the combustion engine with optimum levels of efficiency, it is necessary to decouple the travel speed and engine speed when in motion. This is exactly what various concepts of CVT gearboxes achieve, which in turn necessitate electronic control. The disadvantage of lower engine drag torque in small-volume, supercharged engines during deceleration is also electronically controlled and compensated for by the hydrostatic unit in the drive train. This makes faster, dynamic driving possible while simultaneously reducing fuel consumption.

With regards to the implement hydraulics, the electronification of remotely operated machine functions (e.g. booms) or automated work functions (e.g. road building machines) have already been realised. In earthmoving machines, however, the usually highly-trained driver closes the position control circuit by manually operating the implement hydraulics. The pressure of increased productivity while simultaneously reducing energy consumption also steps up demand on the driver. Implement hydraulics that are optimally adaptable to the driver and the specific work require electronic control. On this basis, assistance functions such as automated levelling are included, for the sustained support and relief of the driver. The cost-efficient realisation of functions such as these using a minimum number of sensors still represents a challenge today.

Many machines require both travel and implement hydraulics at the same time. The hydraulic balancing between the two systems is already at a very high level, and is perceived as a basic prerequisite by manufacturers and users. Electronic control offers a significantly greater degree of balancing flexibility. The machine behaviour then depends heavily on the quality and flexibility of the parameterisation. Further improvements to machine performance will be based on a holistic electronic power management system including the combustion engine in the future. 